\title{
Face Recognition with Modular Two Dimensional PCA under Uncontrolled Illumination Variations
}

\author{
Venkatramaphanikumar S, K. V. Krishna Kishore \\ Department of CS\&E, Vignan's Foundation for Science, Technology and Research, Guntur, India
}

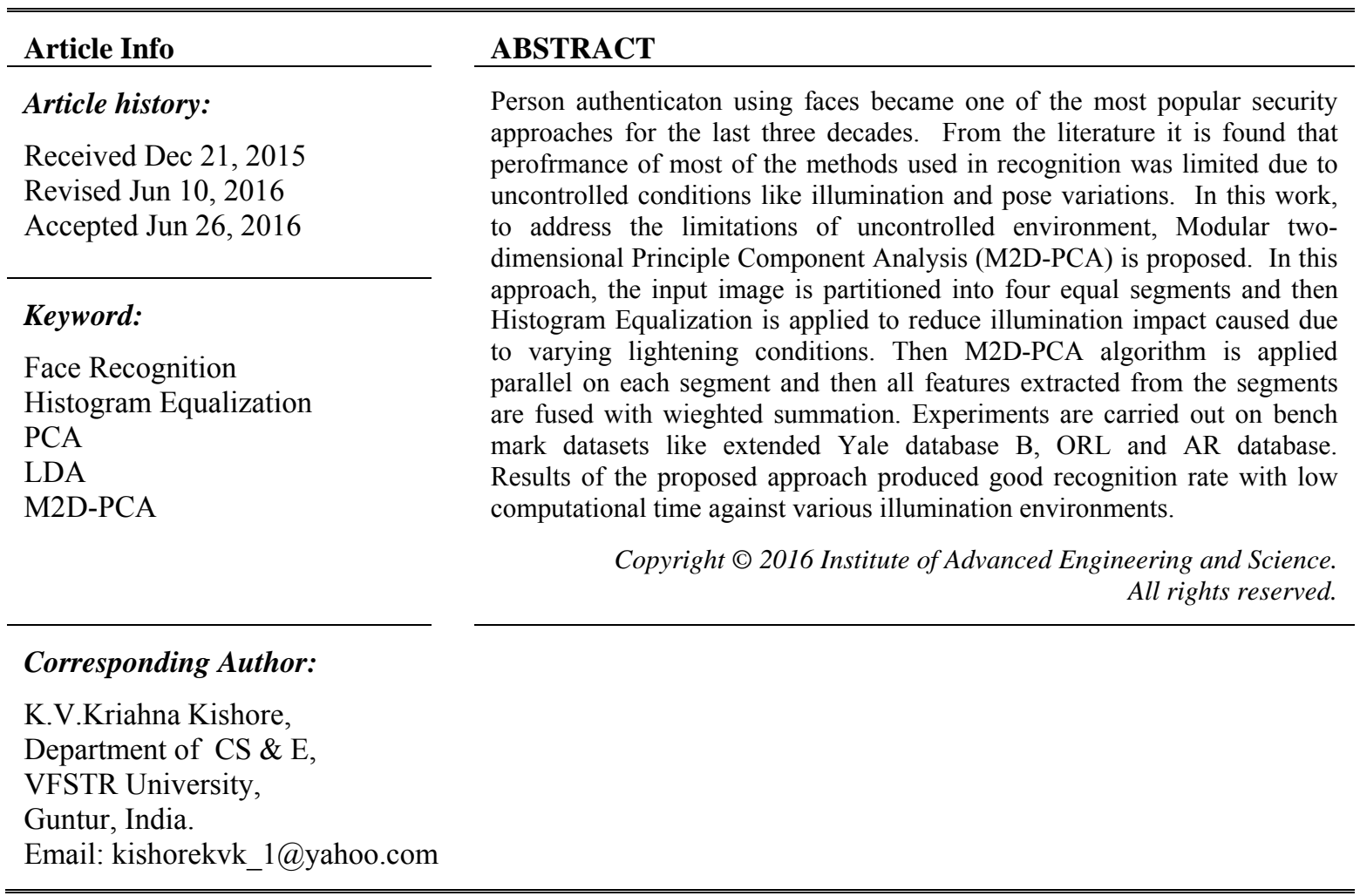

\section{INTRODUCTION}

Person authenticaton using faces is one of the most popular biometric based security systems used in identification and verification. Biometric recognition system using face trait is to recognize person faces against in applications like credit card verification, criminal identification and surveillance systems. Performance of existing systems is low in recognition rate due to multiple variations (Pose and Illumination) $[1,4]$. Face recognition became a difficult problem due to similar shape of the faces and multiple variations among the images of the same person. A biometric application is mainly of three phases; these are Enrolment, Verification and Identification. In the enrolment phase, the system extracts information about the person to be identified by measuring some characteristics. Verification of the person is known as one to one matching and it simply answers whether the person is genuine or not. Identification is one-to-many matching, in which the system selects the best that matches the test sample. There are some key parameters that are used to measure the system performance such as recognition rate and time complexity. There are many approaches in the past that leads to the development of successful face recognition systems. They include: PCA [1], LDA [2], 2D-PCA [3], Face Analysis for Commercial Entities (FACE) [4], and Support Vector Machine (SVM) [5]. Hence, the above methods are successful to a certain extent, but they are limited to the gallery of images that are taken in controlled environments.

This paper introduces a Face Recognition System that works efficiently under uncontrolled environment which overcome the effects of varing illumination. In this work, a weighted fusion approach is used to fuse extracted facial features from modules of face. Objective of this method is defined to enhance the efficiency of the system by extracting the features parallels on segements of faces in recognizing the 
persons under varying illuminations. In this approach first face image has to be portioned into four equal parts. Then pre-processing is done on all partitions independently. Then 2D-PCA algorithm with map reduce approach is used to extract features on each of the individual portions. Score normalization is performed on the extracted feature set to bring all the features into common scale. Proposed model is evaluated on extended Yale face database $\mathrm{B}$ under variant illumination conditions.

\section{PROPOSED METHOD}

\subsection{System Framework}

The main aim of the proposed work is to enhance the efficiency of recognition system for the facial images that are taken under varing illumination conditions.

M2D-PCA is a parallel algorithm with line based local characteristic approach. The architecture of robust face recognition under illumination changes is given in Figure 1. The input image is partitioned into four equal partitions by considering the mean pixel of the image, i.e., LU, LL, RU and RL images. Then, all the four equal segments of the image will undergo Histogram Equalization (HE) phase of mapping as per mapreduce approach. In next phase, M2D-PCA is applied to extract the features. Then, the normalization is applied to get normalized features set. Then the features are fused (reduced) with the weighted summation operation. Nearest neighbour classifier is used for identification of persons. Mapreduce approach is implemented with a cluster of 4 CPUs with 32 GB RAM.

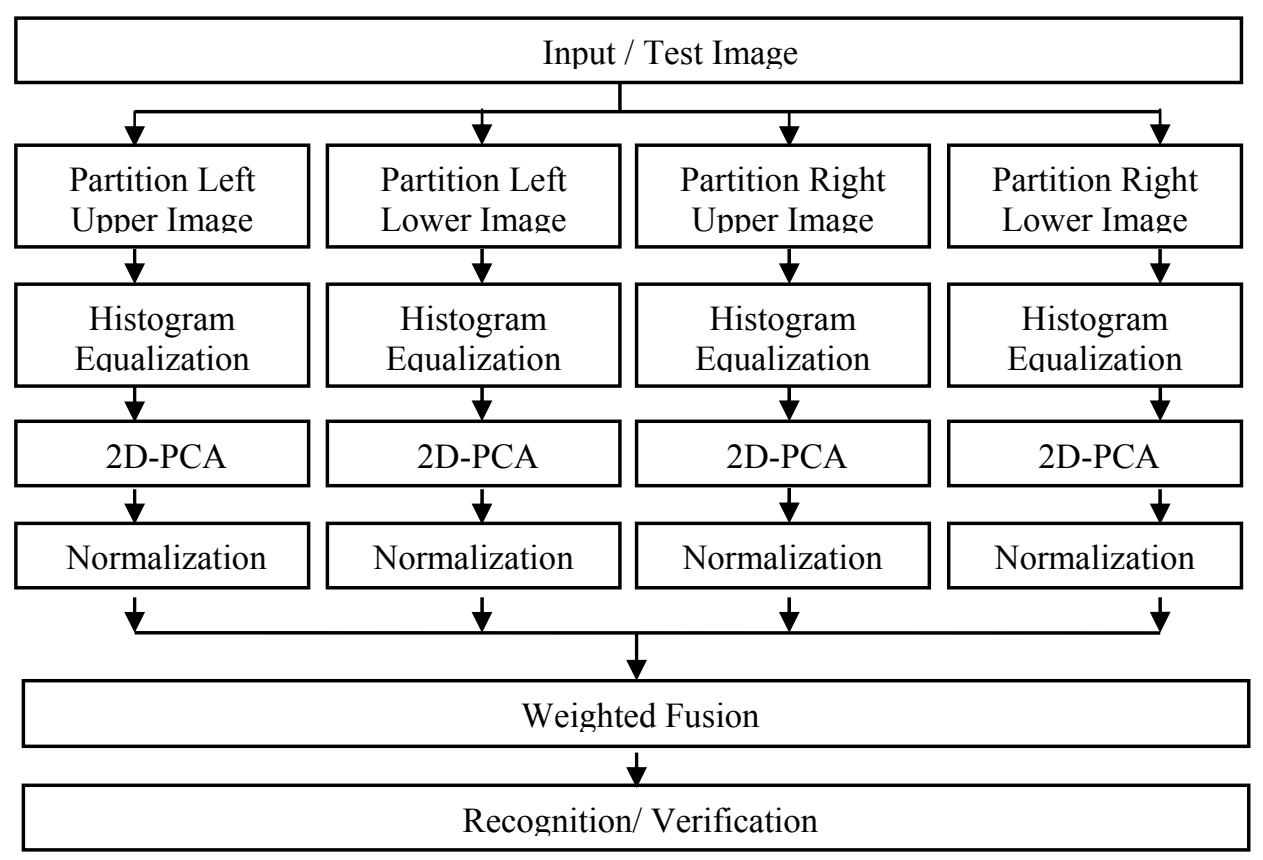

Figure 1. Architecture of the proposed method with map reduce approach

\subsection{Histogram Equalization}

In image processing, objective of the histogram equalization is contrast enhancement of the given image. Histogram Equalization readjusts the original histogram to improve quality of the image by changing pixel gray levels. By the uniform distribution of pixel intensity values, linear cumulative histogram is generated [6]. For an image $\mathrm{I}(\mathrm{x}, \mathrm{y})$ with ' $\mathrm{K}$ ' number of discrete gray values, probability of occurrence of gray level 1 is defined as:

$$
\mathrm{p}(0)=\frac{\mathrm{n}_{\mathrm{k}}}{\mathrm{N}}
$$

where $\mathrm{k}=0,1 \ldots 1-1$ gray scales and $\mathrm{N}$ is the number of pixels of an image. New intensity gray-level of the pixel is defined as $\mathrm{I}_{\text {out. }}$ 


$$
\left.I_{\text {out }}=\sum_{i=0}^{k_{i}-1} \frac{n_{l}}{N V}=\right\rfloor\left((k-1) \sum_{k=0}^{p_{i}-1} p(k)\right)
$$

Output values are in the range of $[0,1]$. To transform the pixel values into the original domain, it will be resized by $\mathrm{K}-1$ value. Figure 2 , shows the facial images before and after histogram equalization.
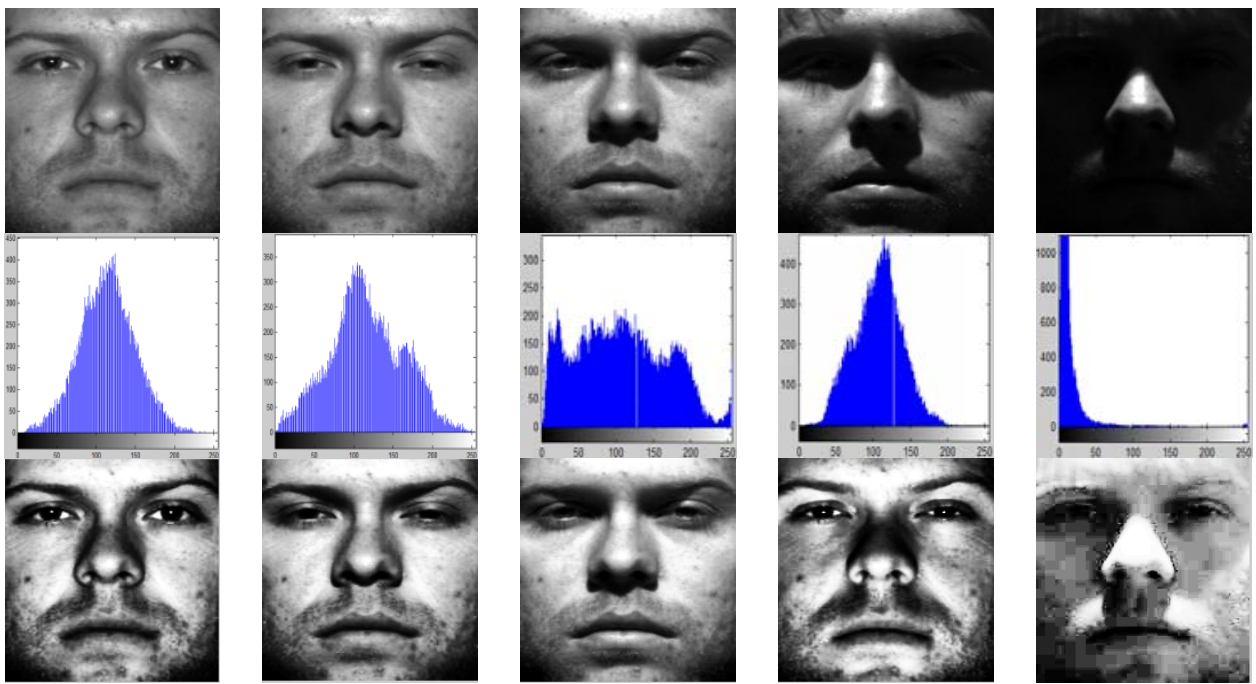

Figure 2. An original image and its histogram and linear histogram equalization

\subsection{M2D-PCA}

\subsubsection{Principle Component Analysis (PCA)}

PCA is one of the classical feature extraction techniques, used to extract the global features which may or may not be naturally understandable. Principal components generated from the eigenvectors are mostly associated with training images. The face images computed with eigenvectors are called as Eigenfaces which appears like ghost faces. Number of images taken in the training set is ' $M$ '. Out of extracted eigenfaces ' $\mathrm{K}$ ' most significant Eigenfaces are used in encoding the variation in face images. Training set with $\mathrm{M}$ samples with each sample size is $\mathrm{N} * \mathrm{~N}$, so total size of matrix is $\mathrm{M} \mathrm{x} \mathrm{N}^{2}$.

1. Average of the training samples is defined as ' $\Psi$ '

$\Psi=(1 / \mathrm{M}) \mathrm{EY}_{1} \mathrm{TI}$

where ' $G$ ' is the image vector.

2. Mean should be subtracted from the training samples and it is defined as $\Phi \mathrm{i}=\Gamma_{\mathrm{i}}-\Psi$

3. Compute Covariance matrix $\mathrm{C}=\mathrm{A}^{\mathrm{T} *} \mathrm{~A}$, where $\mathrm{A}=\left[\Phi_{1}, \ldots, \Phi_{\mathrm{M}}\right]$

4. The eigenvectors of covariance matrix are $\mathrm{U}_{\mathrm{i}}=\mathrm{A} * \mathrm{~V}_{\mathrm{i}}, \mathrm{U}_{\mathrm{i}}$ resemble facial images called as Eigenfaces.

5. Each face image is projected into face space $\Omega_{\mathrm{k}}=\mathrm{U}^{\mathrm{T}}\left(\Gamma_{\mathrm{k}}-\Psi\right)$

6. Then, probe image ' $\Gamma$ ' is projected into subspace to compute a vector ' $\Omega$ ' $\Omega=\mathrm{U}^{\mathrm{T}}(\Gamma-\Psi)$

7. The distance between ' $\Omega$ ' to each class is represented as $\Omega_{\mathrm{k}}=\mathrm{U}^{\mathrm{T}}\left(\Gamma_{\mathrm{k}}-\Psi\right)$ $\epsilon_{\mathrm{k}}^{2}=\left\|\Omega-\Omega_{\mathrm{k}}\right\|^{2}$

8. A distance threshold, $\Theta_{\mathrm{c}}$, is $\Theta_{\mathrm{c}}=1 / 2 \max (\mathrm{j}, \mathrm{k})\left(\left\|\Omega_{\mathrm{j}}-\Omega_{\mathrm{k}}\right\|\right)$

Each image in the training set is a weighted linear combination of basis faces computed. Each eigenvalue represents the amount of variance that has been captured by one component. The number of Principal components obtained is equal to the total number samples in the training set. First principle component holds the highest variance among other principle components. Second principle component holds next to first PC in representing variance.

\subsubsection{Modular Two Dimensional PCA}

Generally in PCA, the 2D image matrices are transformed into one dimensional either column or row vector. Compared to conventional PCA, in 2D-PCA [3],[7],[8] the computation of Covariance matrix is 
simpler and so the time complexity in both training and testing will be reduced. To improve the performance of the modal against variations in illumination and pose, the image is partitioned into four equal segments such as LL, LU, RU and RL as shown in Figure 3.

Let us indicate an image with $\mathrm{m}$ rows and $\mathrm{n}$ columns.

$$
\theta=A X
$$

Here ' $\mathrm{O}$ ' is a projected feature vector of an image $A$. The covariance matrix $\mathrm{G}_{\mathrm{t}}$ is defined as,

$$
G_{t}=E\left[(A-A)^{2}(A-A)\right.
$$

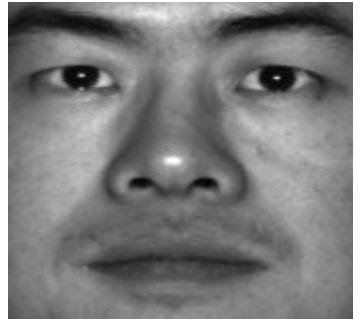

Figure 3(a) Original Image

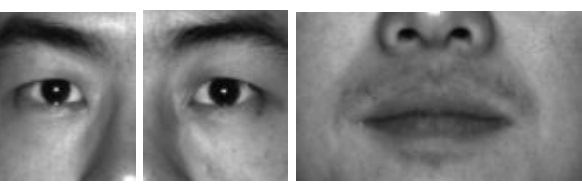

b) Partitioned Images (LU, RU, LL, RL)

Here $\mathrm{E}$ is an expectation and $\overline{\mathrm{A}}$ is the average of the gallery face dataset. Principal component vectors can be shown as, $\mathrm{V}=\left[\mathrm{o}_{1} ; \mathrm{o}_{2} ;: ; ; \mathrm{o}_{\mathrm{S}}\right]$. Where, ' $\mathrm{V}$ ' is denoted as sample image feature vector matrix. Distance among two feature vectors are calculated with the nearest neighborhood classifier. $\mathrm{Vp}=\left[\mathrm{o}(\mathrm{p})_{1}\right.$; $\left.\mathrm{o}(\mathrm{p})_{2} ;:: ; \mathrm{o}(\mathrm{p})_{\mathrm{S}}\right]$ and $\mathrm{Vq}=\left[\mathrm{o}(\mathrm{q})_{1} ; \mathrm{o}(\mathrm{q})_{2} ;:: ; ; \mathrm{o}(\mathrm{q})_{\mathrm{S}}\right]$ is given as,

$$
d\left(p_{p}, p_{q}\right)=\sum_{\xi=1}^{\Sigma}\left\|\alpha_{\xi}^{(p)}-a_{\xi}^{(q)}\right\|
$$

2D-PCA is applied on each modularized face segments and then the feature sets of all modules are needed to be normalized. Normalization process brings the feature set into a common scale. Similarly normalization is applied for test images also. Z Score Normalization is used to generate the similarity score of each modularized face segments of training samples.

$$
\mathrm{Z}=\frac{\Sigma_{\tilde{L}}-\frac{2}{g}}{g}
$$

where $\bar{S}$ represents the arithmetic mean and o represents the standard deviation.

\subsection{Weighted Fusion}

In this phase the features extracted from left upper, right upper, left lower, right lower segments are integrated to improve accuracy of the face recognition. In this work weighted summation method is used to fuse the results of normalized features. In a face image, more discriminative information is available in upper part than lower part. Based on this view, weights are assigned to the modules. Weighted summation is given as

$$
s=\sum_{n=0}^{\infty} w_{n} s_{n}
$$

\section{EXPERIMENTAL RESULTS}

Performance of the proposed method M2D-PCA is evaluated on various standard databases and compared the recognition rates with existing methods. 


\subsection{Face Database}

Extended Yale face database B comprises of 2,414 face images from 38 different subjects having 64 illumination variations are taken for experiment analysis. The Yale database comprised of 10 different individuals from the original database and 28 different individuals of extended Yale database B. Few images from Yale database B are shown in Figure 4.

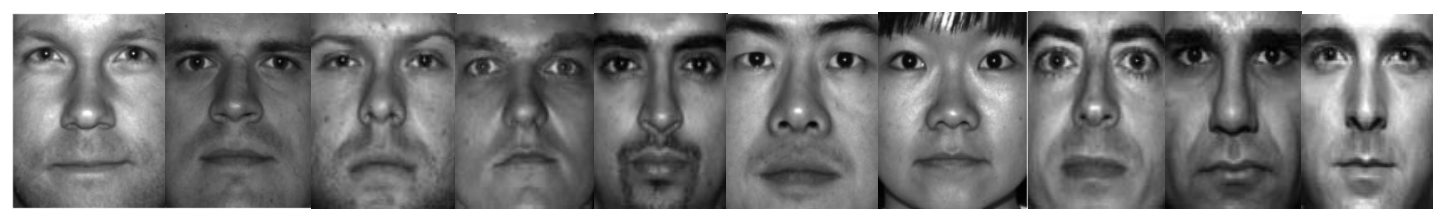

Figure 4. Sample images from Extended Yale database B

ORL Database [9] consists of 40 distinct classes each with 10 samples i.e., 400 images are available. Images are acquired during different times with different illumination and expression variations. ORL also consists of variations in facial expressions. Each sample is of size $92 * 112$.

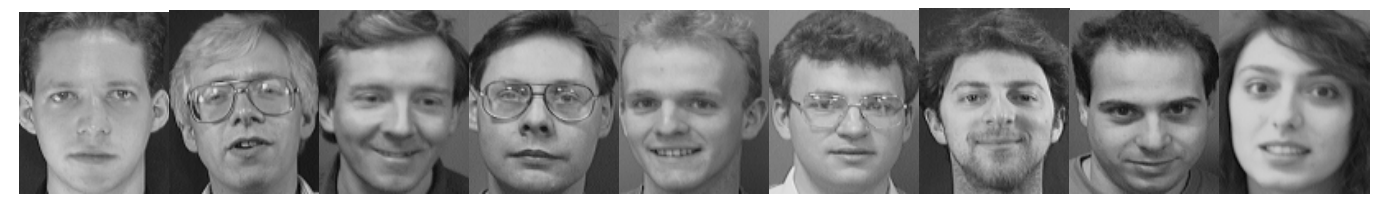

Figure 5. Sample images of ORL Face Database

AR face database [10] consists of 126 distinct classes of unique people, each with 26 samples i.e., 3276 images are there in the database. Images are acquisitioned during different lightening conditions with varied expressions. Each sample is of size $576 * 768$.
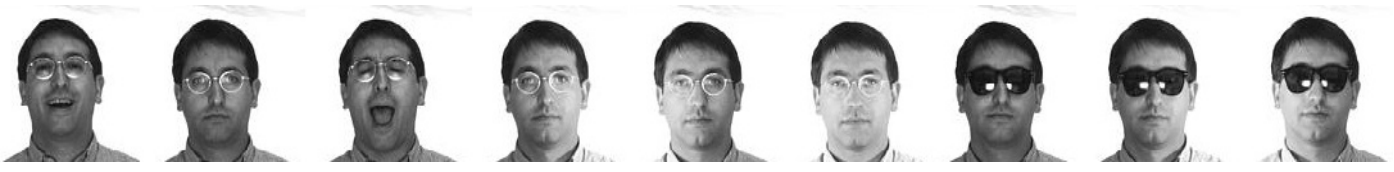

Figure 6. Sample Facial Variation in AR Face Database

\subsection{Performance Evaluation}

To validate the performance of the proposed method, the extended Yale database is partitioned into training set and test set. Here five images per each subject are used as the training set and the remaining fifty-nine images per each subject are used to evaluate the performance of proposed method. Two experiments have been conducted. One is evaluation of performance on whole face with the proposed M2DPCA. Other is evaluation of performance on partitioned segments of faces with the proposed M2D-PCA.

\subsubsection{Experiment 1}

In this experiment analysis, whole image samples from the extended Yale database B are considered during the training and the testing phase. This experimentation mainly carried out to identify whether the proposed M2D-PCA method is robust to illumination variations or not. We considered some other approaches like PCA and LDA for comparative analysis. Recognition rate for these approaches on the raw face database is $24.32 \%$ for PCA and $26.28 \%$ for LDA and $29.21 \%$ for M2D-PCA. After applying histogram equalization on training samples, recognition rates are improved to the rates of $49.21 \%$ for PCA and $56.12 \%$ for LDA and $62.24 \%$ for M2D-PCA. M2D-PCA approach yielded better recognition results compared to 
other methods. This proves that M2D-PCA is robust against all the different illumination variations. The recognition results are given in Table 1.

Table 1. Experimental results for whole images using Yale database B

\begin{tabular}{cccc}
\hline Image Type & PCA & Recognition models \\
& $24.32 \%$ & $26.28 \%$ & M2D-PCA \\
\hline Raw & $49.21 \%$ & $56.12 \%$ & $29.21 \%$ \\
Histogram & & $62.24 \%$ \\
\hline
\end{tabular}

Performance of the proposed method is also evaluated on ORL face Database. Two images per each class are used for training and remaining 8 are used for testing. Results of the proposed method with ORL face database are shown in Table 2.

Table 2. Experimental results for whole images using ORL Face Database

\begin{tabular}{cccc}
\hline Image Type & PCA & Recognition methods \\
& $62.75 \%$ & $71.6 \%$ & M2D-PCA \\
\hline Raw & $85.2 \%$ & $88.74 \%$ & $74.2 \%$ \\
Histogram & & $98.4 \%$ \\
\hline
\end{tabular}

Recognition accuracy of the proposed method is also evaluated on AR Database. Six samples per each class are used for training and remaining 20 samples are used for testing. The performance of the proposed method is shown in Table 3.

Table 3. Experimental results for whole images using AR Face Database

\begin{tabular}{cccc}
\hline Image Type & PCA & Recognition methods \\
& LDA & M2D-PCA \\
\hline Raw & $51.74 \%$ & $74.3 \%$ & $87.3 \%$ \\
Histogram & $69.5 \%$ & $86.25 \%$ & $94.6 \%$ \\
\hline
\end{tabular}

\subsubsection{Experiment 2}

In the second experiment, proposed method is applied on segmented input images such as left upper, left lower, right upper and right lower partitions. Recognition rates of PCA and M2D-PCA methods are computed onYale database B. It is clearly evident that the proposed method is evaluated on segmented ORL face database and performance of the proposed method is $99.24 \%$ with histogram equalised samples where as on raw samples the recognition rate is $69.34 \%$. M2D-PCAmethod is also evaluated on segmented AR face database with 5 training samples and performance of the proposed method is $96.32 \%$ with histogram equalised samples but which is $52.26 \%$ on raw segmented images.

Table 4. Recognition Rates of Proposed method over bench mark databases

\begin{tabular}{ccccccc}
\hline \multirow{2}{*}{ Input Images } & \multicolumn{2}{c}{ ORL Face Database } & \multicolumn{2}{c}{ AR Face Database } & \multicolumn{2}{c}{ Yale database B } \\
& PCA & M2D-PCA & PCA & M2D-PCA & PCA & M2D-PCA \\
\hline Raw Left upper & $38.14 \%$ & $62.15 \%$ & $22.54 \%$ & $42.51 \%$ & $32.16 \%$ & $38.12 \%$ \\
Raw Left lower & $34.18 \%$ & $64.27 \%$ & $23.65 \%$ & $43.56 \%$ & 26.38 & $36.31 \%$ \\
Raw Right upper & $32.48 \%$ & $58.67 \%$ & $21.97 \%$ & $41.29 \%$ & $28.12 \%$ & $42.43 \%$ \\
Raw Right Lower & $33.68 \%$ & $54.19 \%$ & $21.49 \%$ & $40.5 \%$ & $27.53 \%$ & $41.21 \%$ \\
Raw Fusion & $55.28 \%$ & $69.34 \%$ & $32.54 \%$ & $52.26 \%$ & $36.42 \%$ & $48.34 \%$ \\
Histogram Left Upper & $69.34 \%$ & $74.29 \%$ & $45.21 \%$ & $54.12 \%$ & $65.21 \%$ & $87.21 \%$ \\
Histogram Left Lower & $72.35 \%$ & $75.37 \%$ & $43.52 \%$ & $53.68 \%$ & $59.31 \%$ & $79.14 \%$ \\
Histogram Right Upper & $72.52 \%$ & $76.21 \%$ & $42.26 \%$ & $48.69 \%$ & $63.13 \%$ & $89.55 \%$ \\
Histogram Right Lower & $74.91 \%$ & $73.24 \%$ & $44.63 \%$ & $49.57 \%$ & $60.16 \%$ & $83.57 \%$ \\
Histogram Fusion & $82.15 \%$ & $99.24 \%$ & $65.89 \%$ & $96.32 \%$ & $74.34 \%$ & $96.12 \%$ \\
\hline
\end{tabular}

\section{CONCLUSION}

In this paper, the M2D-PCA with map reduce approach is proposed for face recognition under varying illumination environment. It is clearly observable that the recognition results of M2D-PCA is better in comparison with approaches like PCA, LDA for the whole image and segmented images as given above. Results of the proposed method evaluated using four ( 2 x 2), Six (3 x 2) and Eight (4 x 2) equal partitions,

Face Recognition with Modular Two Dimensional PCA under Uncontrolled .... (Venkatramaphanikumar S) 
but the performance is superior with 4 partitions. The performance is evaluated on standard databases such as extended Yale database B, ORL and AR. Future scope of the work is to reduce the time complexity with further optimization in the feature selection.

\section{REFERENCES}

[1] J. F. Pereira, et al., "A robust feature extraction algorithm based on class-Modular Image Principal Component Analysis for face verification," Acoustics, Speech and Signal Processing (ICASSP), 2011 IEEE International Conference on, pp. 1469-1472, 2011.

[2] L. Zhao, et al., "Research on KPCA and NS-LDA Combined Face Recognition," Computational Intelligence and Design (ISCID), 2012 Fifth International Symposium on, vol. 1, pp. 140-143, 2012.

[3] J. Yang, et al., "Two-dimensional PCA: a new approach to appearance-based face representation and recognition," IEEE Transactions on Pattern Analysis and Machine Intelligence, vol/issue: 26(1), pp. 131,137, 2004.

[4] M. D. Marsico, et al., "Robust Face Recognition for Uncontrolled Pose and Illumination Changes," IEEE Transactions on Systems, Man, and Cybernetics: Systems, vol/issue: 43(1), pp. 149,163, 2013.

[5] G. Guodong, et al., "Face recognition by support vector machines," Proceedings of the IEEE International Conference on Automatic Face and Gesture Recognition, pp. 196-201, 2000.

[6] Raju A., et al., "A Comparative Analysis of Histogram Equalization based Techniques for Contrast Enhancement and Brightness Preserving," International Journal of Signal Processing, Image Processing and Pattern Recognition, vol/issue: 6(5), pp. 353-366, 2013.

[7] D. Zhang and Z. H. Zhou, "2DPCA: Two-directional two-dimensional PCA for efficient face representation and recognition," Neurocomputing, vol. 69, pp. 224-231, 2005.

[8] J. Yang and C. Liu, "Horizontal and Vertical 2DPCA-Based Discriminant Analysis for Face Verification on a Large-Scale Database," Information Forensics and Security, IEEE Transactions on, vol/issue: 2(4), pp. 781,792, 2007.

[9] F. Samaria and A. Harter, "Parameterisation of a Stochastic Model for Human Face Identification," Proceedings of 2nd IEEE Workshop on Applications of Computer Vision, Sarasota FL, December 1994.

[10] A. M. Martinez and R. Benavente, "The AR face database," CVC Tech. Report \#24, 1998. 